\title{
Ideología en los contenidos mediáticos: Estudio sobre las series televisivas en España desde la perspectiva de la Cultura de Paz
}

\author{
MHCJ no 5 | Año 2014 \\ Artículo n으 (51) \\ Páginas 35 a 52 \\ mhci.es
}

\author{
Alfonso Cortés González | cortes@uma.es \\ Profesor contratado doctor, Universidad de Málaga
}

Palabras clave

Televisión; ideología; cultura de paz; industrias culturales; opinión pública.

Sumario

1. Introducción

2. Metodología

3. Análisis y resultados preliminares

4. Conclusiones y discusión

5. Bibliografía

\section{Currículum}

Alfonso Cortés González. Vicedecano de Estudiantes y Relaciones con la Empresa de la Facultad de Ciencias de la Comunicación de la Universidad de Málaga. Imparte la asignatura 'comunicación y sociedad' en la facultad de Ciencias de la Comunicación, y 'comunicación de las Administraciones Públicas' en la facultad de Comercio y Gestión.

Coordinador de la asignatura 'Derechos Humanos y medios de comunicación' del máster oficial interuniversitario 'cultura de paz, conflictos, educación y derechos

humanos'.

\section{Resumen}

Casi todos los estudios, al menos desde España, que analizan la influencia mediática sobre la ideología y las cosmovisiones, se centran en el estudio de informativos y discursos políticos. Sin embargo, partimos de la hipótesis de que los contenidos de entretenimiento (cine, shows de televisión, culebrones, videojuegos, etc) pueden incluso tener una influencia mayor en la ideología, actitudes y creencias de las personas que los propios informativos y los discursos políticos. En este artículo se exponen los resultados obtenidos del análisis de las principales series televisivas en España durante 2011, vinculados a una muestra de 2204 ciudadanos, con el objetivo de detectar las correlaciones que existen entre la ideología de la audiencia y la ideología dominante en discurso de la propia serie.

Forma de citar este artículo en las bibliografías

Alfonso Cortés González (2014): “Ideología en los contenidos mediáticos: Estudio sobre las series televisivas en España desde la perspectiva de la Cultura de Paz”, en Miguel Hernández Communication Journal, n5, páginas 35 a 52. Universidad Miguel Hernández, UMH (Elche-Alicante). Recuperado el de de 20

de: http: $/ /$ mhcj.es $/$ index.php?journal $=$ mhcj\&page $=$ article\&op $=$ view\&path $\square=54$ 


\section{Introducción}

Existe un vacío importante a la hora de estudiar y plantear trabajos de investigación sobre ideología y medios de comunicación social, que entre otros factores, reside en que cuando hablamos de ideología en los medios, casi instintivamente ponemos nuestra atención en los contenidos informativos o las líneas editoriales y partidistas de los periódicos. Y no sólo en las noticias o en las declaraciones políticas existe ideología, sino que existe en cualquier manifestación cultural humana, y el contenido mediático de entretenimiento es un claro ejemplo de ello (Durham y Kellner, 2012; Kellner, 1995; Thompson, 2001).

Para enriquecer y avanzar el conocimiento en la materia, queremos poner el foco en los contenidos no informativos. Es decir, queremos fijarnos en aquellos productos audiovisuales que consumimos sin vocación de informarnos, sino para pasar un buen rato o evadirnos (Baran, S.J.; Davis, D.K., 2009).

Por tanto, partimos de la asunción teórica vinculada a los estudios culturales, que enuncia que son más influyentes, desde el punto de vista ideológico y a largo plazo, los contenidos mediáticos que consumimos por diversión frente a los contenidos formativos o informativos (aunque la información también es entretenimiento). Partimos de esa idea que se formuló en la década de los 60 de que el proceso de socialización (y aceptación de ideas) es más eficaz mediante el juego y la diversión, y el gran valor que tiene en nuestra vida en sociedad lo informal frente a lo formal (Giddens, 1964).

Sin embargo, todo producto cultural y mediático, aunque su vocación sea entretener (y no formativa a priori), tiene una dimensión socializadora, es decir, tiene capacidad de educar o mostrar modos de entender la vida. ¿Qué ideología tienen o proyectan los Simpson? ¿Qué modelo cultural fomenta Juego de Tronos? ¿Puede servir el guión de un concurso televisivo para legitimar un modelo político, cultural o social concreto? ¿Existen correlaciones entre la ideología de nuestra serie favorita y nuestra ideología propia? Para tratar de dar respuestas a estas cuestiones, quiero poner en marcha el Observatorio de Ideología en Medios de Comunicación, un proyecto en el que se puedan integrar también nuestros estudiantes de grado y posgrado, y puedan vivir en carne propia lo apasionante que es en realidad la investigación social.

Son obvias y notorias las líneas ideológicas y editoriales de los contenidos con vocación informativa, mientras que estas cuestiones, suelen pasar más desapercibidas para los espectadores en la ficción, ya que la consumimos para pasar un buen rato, evadirnos o desconectarnos de los problemas cotidianos. Decimos que el periódico tal es de derechas, o el periódico cual es de izquierdas, pero no solemos tener conciencia cotidiana en que tal comedia es más conservadora o progresista. 
Además de partir de las teorías sobre medios de comunicación y cultura, hay que manejar también lo que hasta la fecha se ha escrito sobre opinión pública. Hasta los noventa (incluso a comienzos de los 2000) encontramos numerosa literatura sobre opinión pública, persuasión y medios de comunicación. La mayor parte de estas obras son ensayos y reformulación de teorías clásicas como la agenda. Sin embargo es difícil encontrar análisis empíricos. Así, para el background teórico de esta investigación han sido muy útiles los ensayos y teorías de Perloff, McCombs, Lakoff, Van Dijk, Thomson, Giddens, Adorno y Chomsky entre muchos.

En la actualidad, y fruto de la experiencia en campañas de persuasión, sabemos que el discurso mediático influye en la opinión de las personas, pero no sabemos científicamente cómo, de manera concreta, una película o unos dibujos animados contribuyen a crear opiniones e ideas. A largo plazo, sabemos que difundir repetidamente un cierto tipo de mensajes, en un contexto asumible por el público, se pueden potenciar ciertas actitudes personales, clichés e incluso creencias muy sólidas.

Dicho esto, queremos plantear a modo de hipótesis de partida, que algunas comedias televisivas (en las cuáles apreciamos igualdad entre hombres y mujeres, o un trato de naturalidad para con los gays) han contribuido a la igualdad de género o a la normalización de la homosexualidad en la sociedad, mucho más que las campañas de publicidad de los gobiernos y que los propios discursos políticos. Además, estas cuestiones como la igualdad o la naturalización de la sexualidad individual, son poco espectaculares para un informativo, pero sin embargo funcionan muy bien cuando se introducen en el discurso de la ficción, y van creando cotidianidad al mismo tiempo que sirven de ejemplo (pedagogía a través de los medios) en la construcción de la cultura popular. Esto ocurre porque aprendemos por imitación cuando no sentimos la presión del proceso de aprendizaje, y los contenidos mediáticos plantean modelos de relación social imitables o reproducibles (Habermas, 2002; Giddens 2010; Perez Tornero, 1994).

Además, queremos planteamos para el debate académico (sin defender que sea nuestra postura clara) que los mensajes mediáticos que se difunden a través de contenidos de entretenimiento son más persuasivos que cualquier otro tipo de contenidos, porque las barreras psicológicas estas relajadas ya que no atendemos a la posible intención persuasora que pueda existir tras tales contenidos (Perloff, 2003; Simons, 2001). De este modo, el objetivo de este texto es hacer una primera exploración sobre qué tipo de ideología se difunde a través de las series televisivas de ficción, para ir descubriendo cómo estos contenidos afectan (si es que lo hacen) o tienen una relación estrecha con las opiniones y cosmovisiones de los individuos.

Por otro lado, cuando hablamos de ideología, no nos referimos a un conjunto de lemas e ideas cerradas relacionadas con corrientes y partidos políticos concretos (Van Dijk, 1998). En mis estudios e investigaciones anteriores tenía un especial interés en cómo la Cultura de Paz 
podía ser promovida desde las campañas mediáticas que desarrollan las Administraciones Públicas (Cortés, 2007). Ese trabajo me llevó a dos hitos que han sido los desencadenantes de la línea de investigación que abro con este artículo: 1. Las campañas publicitarias institucionales no sirven, en realidad, para influir en las creencias de las personas en un plazo razonable, y 2. Podríamos simplificar las ideologías en solamente dos, bajo la matriz de los parámetros de la teoría de la Cultura de Paz. (Cortés, 2011; Galtung, 1996; Muñoz y Molina, 2009; Fisas, 1998).

Paz y violencia interactúan en seis dimensiones en las cuáles los seres humanos desarrollan sus interrelaciones. Entendemos la violencia como cualquier situación o acto que causa sufrimiento o desequilibrio/desorden a las personas, los animales o el medio ambiente, incluso cuando no es conscientemente intencionado. Los seis planos a los que nos referimos son:

- La naturaleza: seres humanos, animales, plantas, microorganismos, etc. Las ideologías pueden estar sensibilizadas con la naturaleza o no importarle. (Por ejemplo, en la sociedad encontramos el debate político de qué es más importante, el equilibrio del medio ambiente o el consumo energético vinculado con el consumo y un modelo de crecimiento concreto)

- La persona: Necesidades vitales, dignidad, identidad social, individual o étnica. (Ej. Algunos modelos ideológicos se centran en el individuo, otros en el grupo, algunos en las similitudes y otros en las diferencias. Algunas ideologías afirman que cada cual tiene lo que se merece, mientras otras creen que en algunos aspectos el individuo nace en una estructura social que puede marcar su vida entera).

- La sociedad: Género, generación, clase social, nacionalidad o religión. (ej. En algunas sociedades encontramos violencia porque encontramos una acusada desigualdad de género, avalada incluso por las leyes, mientras que en otras, las mujeres han alcanzado igualdad jurídica.

- E1 mundo: Sistema territorial de Estados: países occidentales VS orientales. Norte y sur. Dentro del Estado: capital, sociedad civil, mundo rural o urbano.

- El tiempo: La violencia no sólo es sincrónica, sino que puede pasar de generación a generación o manifestarse en un determinado lapso temporal.

De este modo, se puede interactuar con violencia o paz en cada uno de esos seis planos de manera directa o indirecta, cultura o estructural (Galtung, 1996). Para trazar el modelo ideológico de un individuo hemos atendido no a sus acciones (imposible de medir si no invadimos su intimidad) sino a sus opiniones. Una persona puede considerarse pacífica, y tener compromiso con la igualdad de género, pero al mismo tiempo puede defender, por ejemplo, muchos tipos de violencia estructural o cultural como las corridas de toros o la pena de muerte. Si hacemos una conexión de la teoría de la Cultura de Paz con la teoría política, diríamos que la Cultura de Paz está en el universo conceptual de las ideologías liberadoras y progresistas (teniendo en cuenta lo amplio, complejo y heterogéneo de este grupo de 
ideologías), y la cultura de la violencia lo está con las ideologías autoritarias y conservadoras (igualmente heterogéneas y complejos). Para reducir tal complejidad y simplificar la exposición y transmisión de los resultados, usamos simplemente la distinción única de ideología de paz o ideología de violencia, siendo conscientes de los muchos matices que nos perdemos en esta primera aproximación. En el siguiente epígrafe, explicaremos más detalladamente la metodología que hemos seguido en este trabajo y cómo hemos definido el perfil ideológico de nuestros encuestados.

\section{Metodología}

El objetivo principal de este artículo consiste en detectar si existe correlación entre la ideología que proyectan las series de televisión y la ideología de los telespectadores que siguen tales series, y también en observar si las ideologías de las personas influyen en la selección de las propias series.

Puede parecer banal, pero no lo es. El debate está servido, porque mientras que vulgarmente a una persona que juega a videojuegos con contenido violento como el GTA, se le puede tachar de violenta o que está consumiendo algo reprochable, no se opina lo mismo de una persona que tiene en la estantería de su casa la trilogía de El Padrino. Es decir, por un lado hacemos afirmaciones como si la elección de los productos culturales tuviese una correlación con las conductas y opiniones personales y otras veces no. En este artículo vamos a descubrir qué hay de cierto sobre ello en cuanto al consumo y elección de las series de televisión favoritas.

Para acercarnos al objeto de estudio que hemos definido (estudiar las correlaciones que pudieran existir entre maneras de ver y entender el mundo y el consumo mediático), hemos desplegados y cruzado los datos de tres herramientas de investigación básicas en las Ciencias Sociales:

- Encuesta sobre una muestra de 2204 personas en las provincias de Málaga, Madrid y Barcelona.

- Análisis de contenido de las 9 series de televisión de mayor audiencia, producidas y emitidas en España durante el año 2011.

- Entrevista en profundidad a 57 personas para contrastar, controlar y filtrar los resultados obtenidos en la encuesta.

Hemos encuestado a 2204 personas en las provincias de Málaga, Madrid y Barcelona, tanto de zonas urbanas como rurales y de todos los grupos sociales, siguiendo la división que de la sociedad hacen las Tipologias Mosaic, que es una herramienta utilizada en publicidad y marketing para la segmentación de públicos y mercados. En las encuestas se ha tratado también que el número de encuestados de cada grupo social, sea proporcional a la realidad socio-económica del país. 
El cuestionario está dividido en cuatro bloques de preguntas:

- En el primero se recoge la edad y sexo de la persona encuestada, así como su nivel de estudios.

- En el segundo bloque, las preguntas están orientadas a conocer cuál es el consumo mediático de las personas encuestadas: qué ven en la tele, qué periódicos leen, cuáles son sus programas o series preferidas, etc.

- En el tercer bloque de preguntas, se pretende desvelar qué piensa la persona encuestada sobre cuestiones ideológicas y culturales, así como la igualdad de género, la conveniencia de un sistema fiscal de distribución de rentas, asuntos como el militarismo, cuestiones religiosas, entre otras muchas materias. Para elaborar las preguntas de este bloque se ha seguido una matriz (recreada a partir de los estudios de cultura de paz de Johan Galtung) basada en dos modelos culturales, el modelo cultural de paz, y el modelo cultural de violencia, que se puede complementar con la matriz de valores de un modelo social democrático frente a un modelo social autoritario.

El último bloque de preguntas busca saber el sentido del voto de la persona encuestada (aunque esto no es relevante para el estudio, ya que en España, el voto está muy vinculado a la tradición familiar, y los resultados si atendemos a esta variable, podrían ser muy contradictorios).

Por otro lado, una vez realizadas las encuestas, y atendiendo a las preferencias de nuestros encuestados y a los datos de audiencia, se seleccionaron las serias a analizar. La herramienta utilizada ha sido un análisis de contenido con trazas de análisis crítico del discurso. De cada serie se han analizado tres capítulos como mínimo, y cada análisis ha sido sometido a una segunda revisión ciega para tratar de minimizar la influencia de la propia ideología del investigador o investigadora en los resultados del estudio.

Además, a 57 de los 2204 encuestados los volvimos a reunir para hacerles una entrevista en profundidad sobre los mismos aspectos que el cuestionario, para que nos sirviese de control y filtro a las distorsión de los resultados que siempre nos reportan las encuestas.

Posteriormente, una vez que teníamos los resultados de las encuestas, de los análisis de contenido (sobre las series preferidas), y sobre las entrevistas en profundidad, se cruzaron los datos obtenidos por cada una de estas tres herramientas de investigación, lo que da solidez y coherencia a los resultados que de manera divulgativa y preliminar aquí se presentan.

Nos encontramos, por tanto, con una investigación que desvela datos nuevos sobre el consumo mediático y la influencia ideológica en España, y esperamos que este sea el primero 
de una larga serie de estudios que nos ayuden a entender mejor el mundo, o mejor dicho, entender mejor al ser humano. Esperamos que disfrute este primer informe y que le sea de utilidad académica o profesional. $\mathrm{O}$ atendiendo a las teorías de los usos y gratificaciones, simplemente para su disfrute.

\section{Análisis y resultados preliminares}

De las nueve series estudiadas, seis son dramas, dos comedias, y una ciencia ficción, lo que evita polarizaciones en los resultados que puedan verse influidos por el género, ya que, por ejemplo, en una comedia se pueden emplear recursos narrativos burdos, irónicos o de mal gusto, que en el drama podrían hacer saltar alarmas erróneas en nuestros análisis de contenido. Esto, por otra parte, invita a seguir perfeccionando esta herramienta de investigación para futuros trabajos.

En este epígrafe vamos a exponerles ahora muy resumidamente, por cuestiones de espacio en una publicación de estas características, los resultados preliminares obtenidos por cada una de las series de televisión estudiadas:

\subsection{Amar en tiempos revueltos ${ }^{i}$}

Productora: Diagonal TV

Año de emisión: 2011

Género: Histórico/Drama

Emisora de TV: TVE

Amar en Tiempos Revueltos es la serie más vista de en la franja horaria de sobremesa. Comienza a emitirse en septiembre de 2005, pero no es hasta 2008 cuando alcanza sus mejores resultados de audiencia. Hoy en día podemos decir que después de 6 años de emisión sigue manteniendo a sus casi 3 millones de espectadores cada día. Una serie que ha llegado hasta el $28 \%$ de Share en sus mejores días.

A pesar de una aparente neutralidad de la serie, en su intento de documentación histórica para reflejar la sociedad de la época, podemos vislumbrar una tendencia hacia la Cultura de Paz en sus telespectadores. La obra audiovisual pretende incluir personajes que reflejen los distintos grupos sociales de la época (Guerra Civil Española y primera época del franquismo), pero prestándole más atención al sector democrático prorrepublicano. En este sentido, estos personajes que se declaran en contra del régimen dictatorial y con ideas más progresistas aparecen en todas la temporadas como hilo vertebral en torno a los cuales vienen y van el resto de los personajes que componen el argumento de la obra audiovisual. Además, podemos señalar un tratamiento sobre algunas cuestiones cotidianas (inmigración, homosexualidad, pena de muerte) desde una perspectiva integradora y pacífica. Además, según nuestras encuestas, la audiencia de esta serie vota mayoritariamente a partidos progresistas (sólo un 12,5\% declaró votar a partidos de la derecha). 
Además, casi todos los espectadores de esta serie estiman positivo el pago de impuestos para la construcción social y consideran la inversión en educación mucho más importantes que la inversión en Defensa para los intereses del país.

\subsection{El Barco ${ }^{\text {ii }}$}

Productora: Globomedia

Año de emisión: 2011

Género: Drama/acción

Emisora de TV: Antena 3

La serie El Barco está dirigida a un público joven. La media de audiencia de la primera temporada superó los cuatro millones de espectadores, casi siempre por encima del $20 \%$ de share.

Esta serie proyecta un modelo cultural alejado de principios de igualdad y respeto ya que se hace un uso continuado de argumentos basados en la agresividad y la violencia. Especialmente grave es la exposición que se hace en algunos pasajes de las cuestiones de género: el planteamiento sexista del guión, la construcción psicológica de los personajes y la utilización de la violencia hombre-mujer va totalmente en contra de los principios de igualdad y representan un modelo de conducta violenta en el que incluso se llega a justificar y ejemplificar la violencia de género. En el tratamiento se obvia lo comú, lo colectivo para fortalecer el individualismo y favorece "la cultura del mínimo esfuerzo". Los personajes de la serie reivindican derechos, al mismo tiempo que demuestran una falta de compromiso con las obligaciones y responsabilidades.

En cuanto a la situación en el espectro político de los espectadores cuya serie favorita es El Barco, hemos observado que en los ítems en los que se les preguntaba "¿a qué partido político votas?": las respuestas eran mayoritariamente hacia los dos grandes partidos, PPPSOE, mientras que a la pregunta de cómo se considera ideológicamente, un 18\% se declara por la izquierda, un $36 \%$ se sitúa entre el centro-izquierda y el centro-derecha y un asombroso $45 \%$ se autodefinía ser de derechas, muy de derechas o de ultraderecha.

La ideología que proyecta esta serie tiende fundamentalmente hacia la cultura de la violencia, mientras que su audiencia, aunque con respuestas más moderadas que el discurso de la misma, también tienden hacia la cultura de la violencia en el conjunto de los ítems del cuestionario.

\subsection{Aída $a^{\text {iii }}$}

Productora: Globomedia

Año de emisión: 2011

Género: Comedia

Emisora de TV: Telecinco 
Esta serie es una de las más vistas en España. En su estrenó ostentó un 36\% de share y ha conseguido seguir manteniendo muy buenos índices de audiencias en sus siguientes nueve temporadas. La serie proyecta un modelo cultural más próximo a la Cultura de Paz dato que coincide con los resultados que hemos obtenido de su audiencia.

La serie se desarrolla en un barrio de clase trabajadora de Madrid donde se parodian personajes tipo, fundamentalmente un progresista que vivió el mayo del 68 (pero desde España, lo que le otorga cierta ingenuidad) y un ultraderechista nostálgico del franquismo. Como recurso humorístico, la serie lleva al extremo los tópicos y clichés vinculados con la ideología política de estos dos personajes, encajando perfectamente los propios valores de ambos personajes con los valores de la cultura de paz (en el progresista) y de la cultura de la violencia (en el ultraderechista). Todo ello abordado desde el humor.

En esta serie no apreciamos ninguna tendencia política definida en la intención de voto de su audiencia, como tampoco detectamos autodefiniciones político-ideológicas destacables.

\subsection{4 de abril. La República ${ }^{\text {iv }}$}

Productora: Diagonal TV

Año de emisión: 2011

Género: Histórica/drama

Emisora de TV: TVE

La República ha obtenido un nada desdeñable $17 \%$ de share. La serie cierra su primera temporada por encima de los 3 millones y medio de espectadores. Esta serie ha sido emitida todos los lunes desde enero de 2011 hasta mayo del mismo año. Se han tenido en cuenta cuestiones como el sexismo, la violencia, la paz, el ecologismo, las referencias religiosas, la inmigración y las actitudes racistas.

Esta serie está ambientada en la España de la II República, y se hace eco de los conflictos de la época, estando muy presentes la religión, el machismo y la polarización política. La serie recibió críticas desde algunos medios de comunicación conservadores por considerarla, a su entender, demasiado progresista. La serie proyecta tanto paz como violencia, pero los valores de Cultura de Paz son los que salen favorecidos en el discurso que el guión proyecta.

En el caso de esta serie, la mayor parte de su audiencia se declara de izquierda o centro izquierda en las encuestas, así como sus respuestas en los ítems viran claramente hacia la Cultura de Paz. 


\subsection{Hispania, La Leyenda ${ }^{\underline{\mathrm{V}}}$}

Productora: Bambú Producciones, SL

Año de emisión: 2011

Género: Histórica/drama

Emisora de TV: Antena 3

La audiencia media de esta serie en 2011 (su segunda temporada) se sitúa en una media del $15 \%$ de share.

Esta serie, quizás por el enfoque histórico ambientado en la Hispania romana, presenta un modelo cultural e ideológico donde el hombre es el líder, y la mujer una astuta tejedora del poder entre bambalinas, lo que se vincula a una visión muy conservadora del juego de roles y características culturales propias del hombre y la mujer. En su discurso se representa la gran importancia de ostentar un buen ejército y por tanto la pertinencia de la resolución de problemas mediante la fuerza, lo que hace virar el modelo ideológico de la serie hacia la cultura de la violencia.

En nuestras encuestas, sin embargo, no encontramos una tendencia ideológica definida en su público, ya que encontramos tanto espectadores con gran puntuación en cultura de paz, como espectadores que puntúan alto en cultura de la violencia, lo que neutraliza la posibilidad de definir ideológicamente la audiencia de esta serie.

\subsection{Cuéntame cómo pasó ${ }^{\mathrm{vi}}$}

Productora: Grupo Ganga

Año de emisión: 2011 (desde 2001 en antena)

Género: Drama/historia

Emisora de TV: TVE

La serie Cuéntame tiene como público objetivo personas de mediana edad, a partir de los cuarenta años en adelante. La audiencia media ha sufrido altibajos a lo largo de su emisión, pero podemos afirmar que en 2011 contó con una media de cuatro millones y medio de espectadores y con un share medio superior al $22 \%$, lo que evidencia una cuota de pantalla muy importante.

Esta serie narra las vivencias de una familia y su entorno desde los años 60 hasta la llegada de la Democracia. El modelo sociológico es en realidad de Cultura de Paz a pesar de que nos encontremos con una aproximación histórica a la dictadura franquista realmente edulcorada.

El 100\% de los encuestados afirma creer en la Democracia como el mejor régimen político y que hay que participar en las decisiones políticas. Sin embargo es significativo, atendiendo a 
la respuesta anterios, que el $50 \%$ de los encuestados consideren que la inmigración es perjudicial para el nivel económico del país. A pesar de ello, en el resto de ítems del cuestionario, los valores de Cultura de Paz son los dominantes.

\subsection{Gran Reserva ${ }^{\underline{\mathrm{vii}}}$}

Productora: Bambú Producciones

Año de emisión: 2011

Género: Drama/Thriller

Emisora de TV: TVE

La media de audiencia de la primera temporada superó los tres millones y medio de espectadores, casi siempre cerca al $20 \%$ de share. El primer pase del primer capítulo de la serie Gran Reserva se emitió el 15 de Abril de 2010 en horario de prime time de TVE1, obteniendo un $20,9 \%$ de cuota de pantalla.

Estamos ante un serial de amores, venganzas y rencillas. El marco de la serie es el mundo vinícola de La Rioja. Compatibiliza el campo, la ciudad, las tinajas y las fiestas, sin resaltar en ningún momento penurias ni miserias.

La serie Gran Reserva proyecta un modelo cultural alejado de principios de igualdad y respeto, haciendo un uso continuado de argumentos basados en la maldad y la violencia. Las diferencias sociales están especialmente latentes en la serie. El planteamiento sexista del guión y la construcción psicológica de los personajes viran claramente hacia la cultura de la violencia. Sin embargo la audiencia tiende hacia la Cultura de Paz.

\subsection{Los Protegidos $\frac{\text { viii }}{}$}

Productora: Ida y vuelta

Año de emisión: 2011

Género: ciencia ficción

Emisora de TV: Antena 3

Es una serie situada en Prime Time y catalogada como una serie para todos los públicos, dirigida a las familias. Conserva un puesto importante dentro del panorama televisivo, con un $18,2 \%$ de share de media en la primera temporada, esto supone una media de 3.360 .000 de espectadores, lo que invitó a la producción de una segunda temporada también caracterizada por su éxito, aunque viéndose algo disminuida con un 15.09\% de share.

Con los Protegidos nos encontramos con un drama fantástico donde niños con poderes mágicos, por diferentes circunstancias, se quedan solos y acaban uniéndose formando una nueva familia para aparentar normalidad y sobrevivir (importancia de pertenencia a un 
grupo). En la serie se pueden apreciar una tendencia hacia estereotipos machistas, aunque es neutral en cuanto a Cultura de Paz o de violencia se refiere.

Con respecto al uso de la violencia directa en la narración, destacamos que el objetivo principal de un personaje es matar a su hermano por haberlo abandonado cuando eran pequeños

La audiencia de esta serie tiende claramente hacia la Cultura de Paz (en casi todos los ítems las respuestas fueron favorables a la Cultura de Paz), y se declaran en su mayoría progresistas.

\subsection{La que se avecina ${ }^{\mathrm{ix}}$}

Productora: Mediaset

Año de emisión: 2011

Género: Comedia

Emisora de TV: Telecinco

En esta serie se narran las vivencias de una comunidad de vecinos, donde todos se llevan mal. La serie obtuvo en su estreno un $28,8 \%$ de share y ha gozado de grandes audiencias en todas sus temporadas.

El sexismo y la violencia son los recursos más frecuentes sobre los que los guionistas buscan el humor y lo divertido. También encontramos una ligera tendencia al machismo en cuanto al tratamiento narrativo del hombre y la mujer. Los perfiles psicológicos en esta serie están trazados a grandes rasgos, siendo la obsesión de los hombres el sexo, y la obsesión de las mujeres el dinero.

La serie aunque neutral, quizás por ser una comedia, vira ligeramente hacia cultura de la violencia, y sus espectadores también neutral pero virando ligeramente hacia Cultura de Paz.

\section{Conclusiones y discusión}

Una vez que se han analizado las 9 series de televisión de mayor audiencia entre la muestra social con la que hemos trabajado, podemos ordenar las ideas para obtener las conclusiones de este estudio y plantear algunos motivos de discusión y debate. A continuación mostramos la tabla en la que encuentran cada una de las series analizadas para este artículo y la tendencia ideológica que fomentan en su discurso, en paralelo a la tendencia ideológica del público que las consume:

Tabla 1

Ideología en series españolas e ideología de su audiencia ${ }^{\mathrm{x}}$

\begin{tabular}{|l|l|l|}
\hline Serie de TV & Ideología de la serie & Ideología de la audiencia
\end{tabular}




\begin{tabular}{|l|l|l|}
\hline Amar en tiempos revueltos & Cultura de Paz & Cultura de paz \\
\hline El barco & Cultura de violencia & Neutral-cultura de violencia \\
\hline Aída & Cultura de Paz & Neutral \\
\hline 14 de abril La República & Cultura de Paz & Cultura de Paz \\
\hline Hispania & Cultura de violencia & Neutral \\
\hline Cuéntame cómo paso & Cultura de Paz & Cultura de Paz \\
\hline Gran Reserva & Cultura de violencia & Neutral-Cultura de Paz \\
\hline Los Protegidos & Neutral & Cultura de Paz \\
\hline La que se avecina & Neutral-cultura de violencia & Neutral \\
\hline
\end{tabular}

Fuente: Elaboración propia

Lo primero que observamos una vez ordenados los datos, es que la mitad de las series estudiadas tienden a un modelo ideológico de Cultura de Paz, mientras que la otra mitad tiende a la cultura de la violencia. Una sola serie la hemos calificado como neutral, ya que en el análisis de contenido, aún encontrando en su discurso aspectos de paz y de violencia, el valor que nos daba al sumar los aspectos de Cultura de Paz y restar los aspectos de cultura de violencia era cero.

Gráfico 1

Ideología en las series de producción española 2011

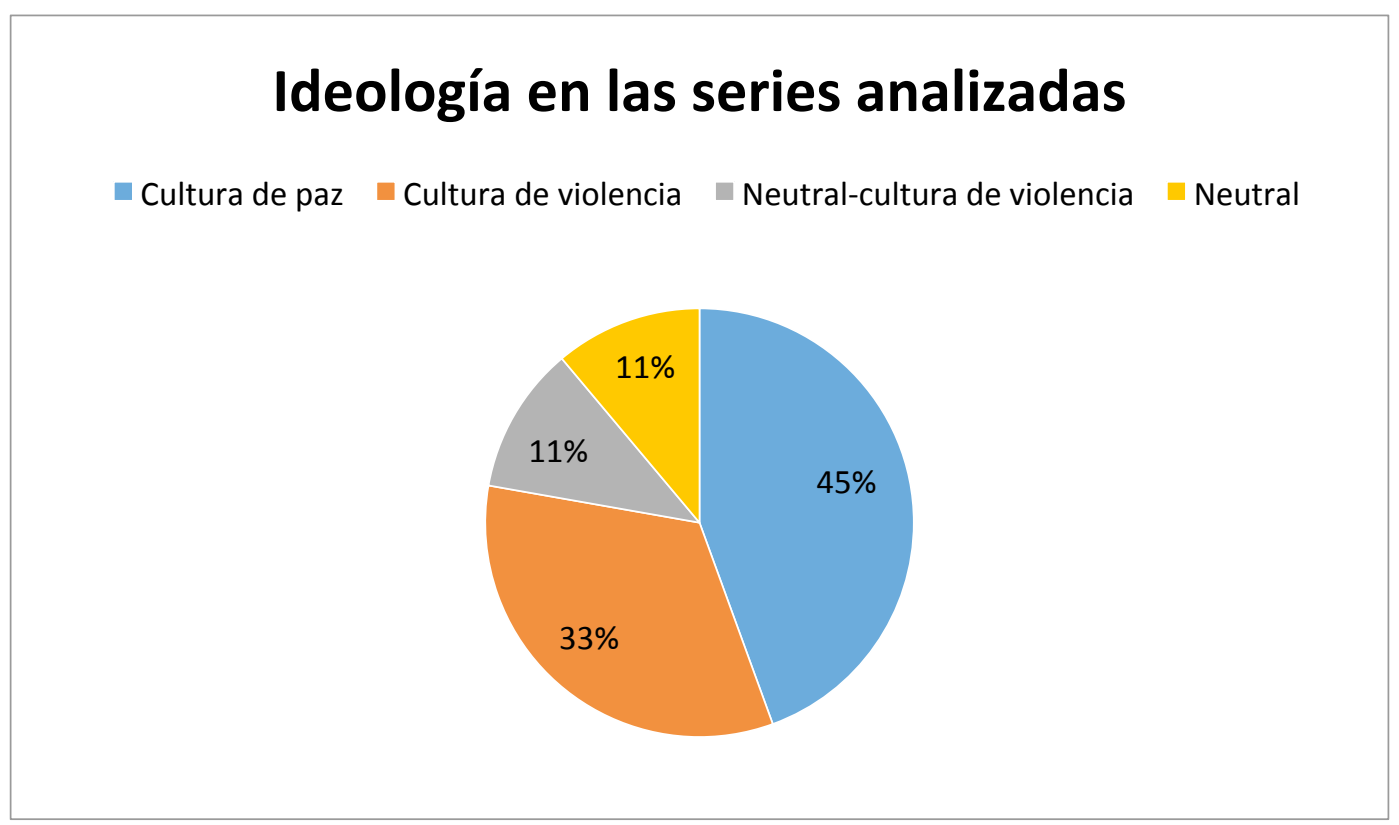

Fuente: elaboración propia

En cuanto a la ideología de las audiencias (del público vinculado a cada serie), los porcentajes de Cultura de Paz son similares a los de las series de TV (el 45\% de la población estudiada) pero sin embargo, el resultado global de los espectadores de series de cultura de la violencia 
viran a la neutralidad. Esto puede deberse a un enmascaramiento, por parte del encuestado, de ciertas posturas u opiniones radicales ante el encuestador. Debemos tener en cuenta que las personas suelen ocultar aquellas opiniones, que aunque las sientan como verdaderas, las consideran políticamente incorrectas.

Gráfico 2

\section{Ideología de las audiencias}

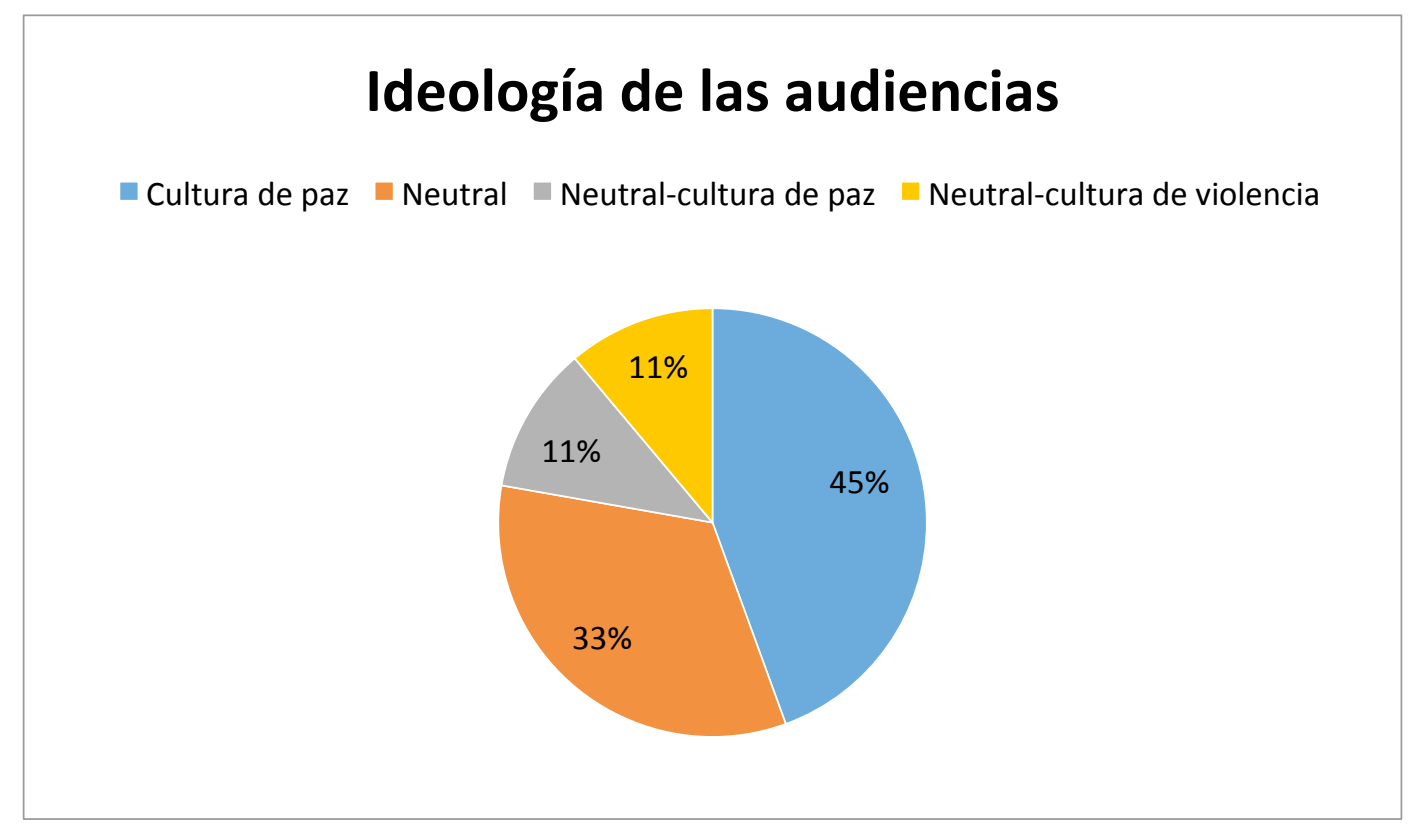

Fuente: Elaboración propia

Lo que si podemos afirmar, sin tener en cuenta juicios de valor que se encuentren fuera de los datos obtenidos, es que existe una vinculación directa de la ideología de las series y de su audiencia cuando el modelo ideológico es de Cultura de Paz. No obstante, cuando observamos series cuyo modelo ideológico es de cultura de violencia, su público nos aparece como neutral, lo que en realidad está indicando que nos encontramos ante una audiencia mucho más heterogénea, lo que la desdibuja ideológicamente, aunque los individuos estuviesen polarizados ideológicamente (no nos encontramos ante ese caso).

Esto nos puede llevar a pensar que los contenidos de cultura de la violencia podrían ser más ambivalentes y comerciales para las emisoras de televisión porque no el público no los rechazaría en función de sus aspectos ideológicos. Llevándonos este asunto a las etiquetas políticas comunes, podemos afirmar que podría existir una tendencia importante en que las personas consideradas progresistas, no seleccionan las series en función de su ideología, mientras que las personas consideradas conservadoras, sí discriminan unas series frente a otras en función de su modelo ideológico.

Estas cuestiones las hemos confirmado en las entrevistas en profundidad, donde las personas progresistas, soportaban perfectamente series televisavas con personajes y discursos 
conservadores, y lo entendían como parte del guión, mientras que gran parte del público conservador, decidía directamente no ver una determinada serie de televisión porque la consideraba muy liberal o directamente, y cito textualmente una respuesta: "no veo esa serie porque es de rojos y maricones".

Por tanto, se puede afirmar, no en función de esta respuesta que podría ser aislada, sino en el conjunto de la muestra estudiada, que las personas cuyo modelo ideológico tiende a la cultura de paz y al progresismo son más abiertas y complejas a la hora de consumir productos cultural, concretamente series de televisión.

Sin embargo, esta conclusión que hemos obtenido a partir de las series, que nos demuestra que el público progresista es más abierto a la hora de consumir series de televisión, puede no darse en otro tipo de productos culturales, como pueden ser los informativos (los periódicos, por ejemplo, tienen un público con un perfil ideológico claro). Esto da una respuesta parcial al objetivo que nos marcamos en detectar si existe una correlación directa entre la ideología que proyectas las series de televisión y la ideología de sus espectadores. En cualquier caso, esta discusión nos abre un nuevo interrogante y nos invita a continuar las investigaciones en este sentido.

En la introducción poníamos el ejemplo de que popularmente hay gente que estima como violentos a aquellas personas que se divierten con videojuegos violentos, mientras no hacen el mismo juicio de valor con una persona apasionada del cine negro. Al menos parcialmente lo que hemos evidenciado en este estudio es que la elección de productos culturales violentos no está directamente vinculada con la ideología del espectador que los consume, aunque sorprendentemente no hemos detectado el mismo fenómeno en el caso contrario.

De cualquier forma, lo que sí queda constatado y nos permite continuar esta línea de investigación, es que existen correlaciones aún no lo suficientemente estudiadas entre la ideología que proyectan los productos culturales y su influencia en la sociedad o bien sobre la influencia de la sociedad en la construcción ideológica de los productos culturales. En cualquier caso, y sea como fuere, queda selva por explorar. 


\section{Bibliografía}

BARAN, STANLEY J. AND DAVIS, DENNIS K. (2009). Mass communication theory : foundations, ferment, and future (6th ed. ed.). Boston, MA: Wadsworth.

Cortés GonzÁlez, A. (2007): Cultura de Pazy Publicidad Institucional. El Estado en el fomento de la Cultura de Paz a través de la publicidad televisiva. Jaén, SP: Alcalá Grupo Editorial.

CORTÉS GONZÁLEZ, A. (2011): "La publicidad institucional en España: una década en perspectiva”, Razón y Palabra, Volume 75. Monterrey, MX: Tecnológico de Monterrey.

Durham, M. AND Kellner, D. (2012): Media and cultural studies keyworks. Ney Jersey, NJ: WileyBlackwell.

FISAS, V. (1998): Cultura de Pary gestión de conflictos. Barcelona, SP: Icaria.

Galtung, J. (1996): Peace by Peaceful Means. London, UK: SAGE Publications.

GIDDENS, A. (1964): "Notes on the concepts of play and leisure", The Sociological Review, Volume 12, Issue 1, pages 73-89. New Jersey, NJ: Wiley.

GidDENS, A. (2010): Sociología. Madrid, SP: Alianza Editorial

KELLNER, D. (1995): Media culture: cultural studies, identity and politicsbetween the modern and the postmodern. London, UK: Routledge.

LAkoff, G. (2002): Moral Politics: How Liberals and Conservatives Think. Chicago, IL: Chicago University Press.

Muñoz, F. And Molina, B. (ed) (2009): Pax Orbis. Complejidad y conflictividad de la paz. Granada, SP: University of Granada Press.

PÉrez Tornero, J.M. (1994): El desafío educativo de la televisión. Barcelona, SP: Paidós 
Perloff, R.M. (2003): The Dynamics of Persuasion. Communication and Attitudes in the 21st Century. London, UK: Lawrence Erlbaum Associates.

Simons, H. W. (2001): Persuasion in Society. London, UK: SAGE Publications.

STEVEnSON, N. (2002): Understandig media cultures. London, UK: SAGE Publications.

Thompson, J.B. (1995): The media and modernity. A social theory of the media. Standford, CA: Standford university Press,

VAN DiJK, T. (1998): Ideología. Barcelona, SP: Gedisa Editorial

\section{Alfonso Cortés González}

Facultad de Ciencias de la Comunicación

Universidad de Málaga

C/León Tolstoi, 4

29071 Málaga

España

Tel. 952137318

Fax. 952133284

cortes@uma.es

www.alfonsocortes.com 


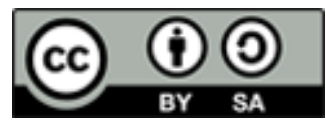

Licencia Creative Commons

Miguel Hernández Communication Journal

mhcj.es

\section{Forma de citar este artículo en las bibliografías}

Alfonso Cortés González (2014): "Ideología en los contenidos mediáticos: Estudio sobre las series televisivas en España desde la perspectiva de la Cultura de Paz”, en Miguel Hernández Communication Journal, n5, páginas 35 a 52. Universidad Miguel Hernández, UMH (ElcheAlicante). Recuperado el__ de de 20

de: http:/ / mhcj.es/index.php?journal $=$ mhcj\&page $=$ article\&op $=$ view\&path $\rrbracket=54$

\footnotetext{
i Análisis de contenido a cargo de Santiago Sánchez de Castro y Leticia Fuentes Alaminos

ii Análisis de contenido a cargo de Ana Isabel Morales Campoy y Andrés Calvo Serrano

iii Análisis de contenido a cargo de Ana Isabel Morales Campoy y Andrés Calvo Serrano

iv Análisis de contenido a cargo de Emilia Luque Bueno y Ana Isabel Morales Campoy

v Análisis de contenido a cargo de Javier López Aranda y David Polo Serrano

vi Análisis de contenido a cargo de Hermes Cañizares Alzórriz y Amanda Serrano Barton

vii Análisis de contenido a cargo de Pilar Tejero Durán y David Polo Serrano

viii Análisis de contenido a cargo de Marta Lupión Lozano y Rosario Serrano

ix Análisis de contenido a cargo de Marina Guadix y David Polo Serrano
}

x Explicación para entender la tabla: Utilizamos la etiqueta de Cultura de Paz cuando la mayor parte de los ítems del análisis de contenido o de las respuestas de las personas (en la encuesta se utiliza la escala de Likert), están vinculados con la Cultura de Paz, dando al menos un resultado de +3 . Cultura de violencia cuando la mayor parte de los ítems del análisis de contenido o de las respuestas de las personas están vinculados con la cultura de la violencia, dando al menos un resultado de -3. Utilizamos neutral cuando el valor de restar los ítems violentos a los de paz es 0. Neutral-Cultura de Paz lo utilizamos cuando el valor de la suma/resta de los ítems de cultura de paz o cultura de violencia es entre +1 o +2 . Neutral-cultura de violencia lo empleamos cuando el valor de la suma/resta de los ítems de cultura de paz o cultura de violencia es entre -1 o -2 